\title{
Photonic integrated technology for space applications
}

Leili A. Shiramin, A. Abbasi, H. Mohammadhoseini, W. Philibert, S. Roemer, et al.

Leili A. Shiramin, A. Abbasi, H. Mohammadhoseini, W. Philibert, S. Roemer, N. Karafolas, P. Van Loock, M. Cuzzola, B. Desoete, "Photonic integrated technology for space applications," Proc. SPIE 11852, International Conference on Space Optics - ICSO 2020, 118523R (11 June 2021); doi: $10.1117 / 12.2599620$

SPIE Event: International Conference on Space Optics - ICSO 2021, 2021, Online Only 


\section{International Conference on Space Optics-ICSO 2020}

Virtual Conference

30 March-2 April 2021

Edited by Bruno Cugny, Zoran Sodnik, and Nikos Karafolas
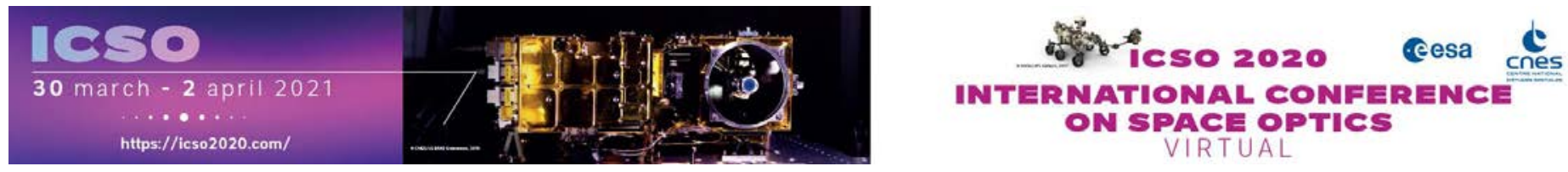

\section{Photonic integrated technology for Space applications}

\section{Cesa isonematang lecnes}




\title{
Photonic integrated technology for Space applications
}

\author{
L. Shiramin, A. Abbasi, H. Mohammadhoseini, W. Philibert, S. Roemer, N. Karafolas, P. Van \\ Loock, M. Cuzzola, B. Desoete \\ stephan.roemer@antwerpspace.be \\ Antwerp Space|an OHB company, Berkenrodelei 33, B-2660 Antwerp, Belgium
}

\begin{abstract}
Space stringent requirements in one hand and huge demand for the high performance, low cost modules on the other hand push the Space technologies toward more compact and integrated solutions. This is a trend inherited from Electronic world being transformed from a transistor level boards to more advance and functionalized ICs with million components integrated inside. Photonic Integrated Circuit (PIC) based optical devices are dominating the terrestrial domain in medical facilities, datacenters and civil infrastructures. There is no doubt, in order to reduce the AIT cost and increase the performance PICs are the recommended solution for the future needs including Space Market. There are obvious advantages to use PICs instead of discrete components which could be categorized in three levels: performance, $\mathrm{SWaP}$ and cost. With respect to the performance, integration potentially means lower coupling loss between components. For microwave photonic application, any optical loss in $\mathrm{dB}$ is translated to $2 \mathrm{~dB}$ loss in the electrical signal. Reduced size and weight is the distinct advantage of the integration where multiple optical channels and components are designed and manufactured in small form factor with potentially improved power consumption. This reduces the packaging, qualification and assembly costs.
\end{abstract}

Our recent activities regarding using PIC for Space is aiming for more compact and high performance modules. The developments include Frequency conversion, Beam forming and Lantern receiver for inter-satellite links.

Keywords: Photonic integrated circuits, frequency conversion, beam forming, lantern receiver

\section{INTRODUCTION}

Photonic based systems have been demonstrated to exhibit better performance compared to conventional RF systems $[1,2]$. That is the motivation for us to focus on new technology for space applications with the aim to offer better performance and lower cost to the satellite communication systems. Some of our research activities are sponsored by ESA ARTES program and some by EU Commission where we address the call LC-SPACE-14-TEC-2018-2019 on Earth observation Technologies.

Whenever individual components are used in an optical system, the connection between them are via a fiber or chip-tochip connections. From the packaging and mechanical/thermal robustness point of views, these transitions introduce the weak points on the final equipment assembly and add coupling loss to the system. By integrating more components on a single die, not only these transition numbers are reduced but also another level of compactness is achieved. Furthermore, by each screening and qualification test on the final packaged device, two or more optical components will be tested which saves substantial amount of the qualification cost later on.

The under-development products are a collection of photonic integrated circuit building blocks for microwave applications. They integrate high performance optical components (laser, modulator, amplifier, detector, etc.) into a single chip. In this paper, we review their application in three domains of frequency conversion, beam forming and lantern receiver. 


\section{ELECTRO-PHOTONIC FREQUENCY CONVERTER}

The basic concept of microwave photonic frequency conversion is to imprint a RF input signal on an optical carrier using an optical modulator, such as a Mach-Zehnder modulator (MZM). The RF signal can then be mixed with a local oscillator (LO) in the optical domain, after which it is converted back to an electrical output signal using a photodiode [3]. In this work, $50 \mathrm{GHz}$ range signal is down converted to $20 \mathrm{GHz}$ range signal using optical link which enables broadband operation and improved SWaP compared to conventional RF based down converters. The same building blocks and architecture developed under EPFC can also be used for the LO signal distribution over optical fiber and data transducer application. In Figure 1 and 2, the architecture of the EPFC, LOD and DT, is depicted.

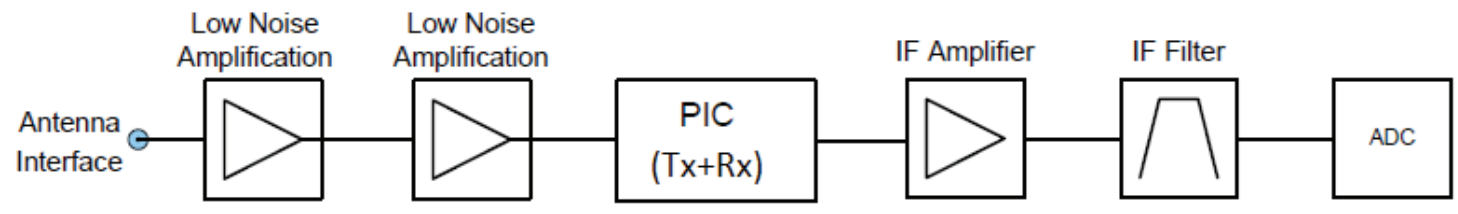

Figure 1. Block diagram showing the RF front-end and PIC in EPFC
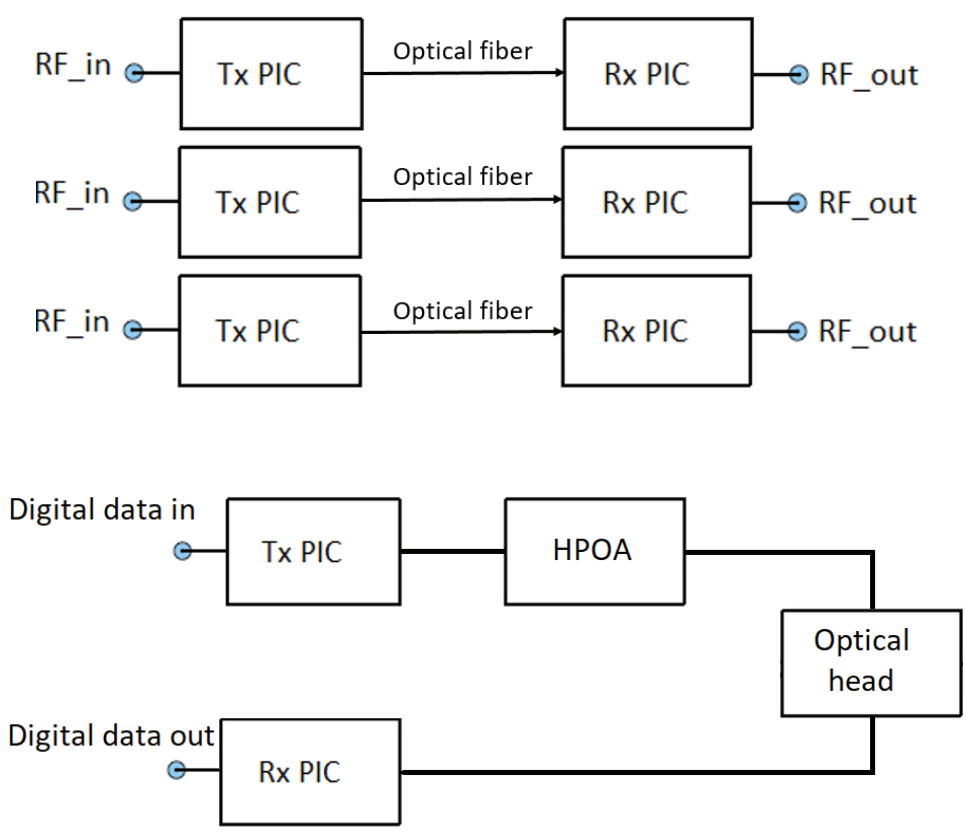

Figure 2. Top: conceptual schematic of LOD (LNAs can also be included). Bot: Data transceiver

The PIC consists of Tx and Rx photonic ICs where we have integrated DFB laser and IQ modulator on the Tx chip and integrated SOA and PD on the Rx chip. Since the PIC is sensitive to the thermal variation, the PIC temperature should be controlled at $20^{\circ} \mathrm{C}$ (PIC is designed for this temperature) regardless of the environmental temperature variation over $15^{\circ} \mathrm{C}$ to $65{ }^{\circ} \mathrm{C}$. This is done via a TEC module underneath the PICs. A change in temperature will lead to e.g. a wavelength change of the laser or its output power variation. The performance of some of other active optical components is also deteriorated by temperature variation. Therefore, the PPIC temperature shall be tightly controlled via the fast feedback circuitry. These active components will be controlled via the feedback loop at specific set points. Biasing of each component requires a precise feedback system and stable circuitry. 
The PICs (photonic integrated circuits) will hermetically be packaged (hereafter called PPIC) and thermally controlled with thermal components inside the PPIC. The PPIC will be positioned in the package to be in the center of the module surrounded by control electronic components.

Each active component in the Frequency converter module needs a supply voltage and current. The RF components, laser, SOA, monitor PDs (for monitoring the power level of laser and IQM) and the high-speed PD require a fixed voltage or current value to be operational but the IQM and thermal components require controlled bias condition that should be adjusted based on the received feedback to the control electronic circuit.

Table 1: EPFC main specifications

\begin{tabular}{|l|l|l|}
\hline Parameter & Value & Unit \\
\hline Frequency range & $\begin{array}{l}\text { RF: } 47.2-50.2 \\
\text { LO: up to } 30 \\
\text { IF: up to } 20.2\end{array}$ & $\mathrm{GHz}$ \\
\hline RF link gain & $\sim 45 \mathrm{~dB}$ & $\mathrm{~dB}$ \\
\hline Noise Figure & $\sim 3 \mathrm{~dB}$ & $\mathrm{~dB}$ \\
\hline $\begin{array}{l}\text { Input optical power } \\
\text { Of integrated laser } \\
\text { with IQM }\end{array}$ & 10 & $\mathrm{~mW}$ \\
\hline Wavelength & 1.5 & \\
\hline Modulator dc $\mathrm{V}_{\pi}$ & $<2$ & $\mathrm{um}$ \\
\hline PD Responsivity & $0.8 \mathrm{~A} / \mathrm{W}$ & $\mathrm{V}$ \\
\hline Fibre connector & $\begin{array}{l}\text { MINI AVIM } \\
\text { PM/APC }\end{array}$ & $\mathrm{A} / \mathrm{W}$ \\
\hline Polarisation sensitivity & TE mode is maintained in the whole chain & \\
\hline $\begin{array}{l}\text { Input/output } \\
\text { impedance }\end{array}$ & 50 ohm & ohm \\
\hline RF interface & GPPO/SMPM & $\mathrm{W}$ \\
\hline Power consumption & $\begin{array}{l}\left.\text { Typical (at ambient up to } 20^{\circ} \mathrm{C}\right) \sim 5 \mathrm{~W} \\
\left.\text { Max (at ambient } 65^{\circ} \mathrm{C}\right) \sim 8 \mathrm{~W}\end{array}$ & years \\
\hline Mission lifetime & 15 & $\mathrm{~mm}$ \\
\hline $\begin{array}{l}\text { Dimensions } \\
\text { PPIC+board+housing } \\
\text { Length } 130 \\
\text { Height } 100 \\
\text { Depth } 33 \\
<0.5\end{array}$ & $\mathrm{Kg}$ \\
\hline $\begin{array}{l}\text { Mass including } \\
\text { mechanical housing } \\
\text { and control electronics }\end{array}$ & \begin{tabular}{l} 
\\
\hline
\end{tabular} & \\
\hline
\end{tabular}

After assembly, the PPIC will undergo the environmental testing for demonstrating its space compliance. The other EEE devices in the EM (current phase) are space grade components.

The anticipated product package in the EM is shown in Figure 3. In the FM phase, the die version of input RF amplifiers will be used and integrated inside the housing. 


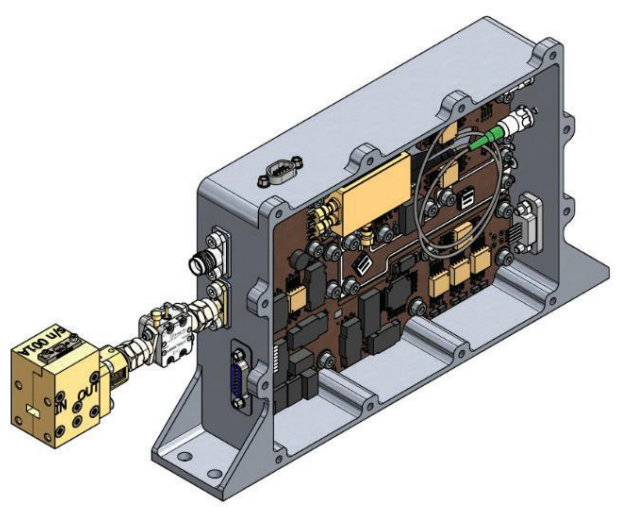

Figure 3. EPFC housing with PPIC, control electronic and RF components

\section{OPTICAL BEAMFORMING}

Microwave remote sensing on Satellite missions plays a key role in monitoring and understanding earth changes over time. For the earth observation, an advance SAR mode has been introduced called scan-on-receive (SCORE). The SCORE principle is based on the illumination of a wide swath. Several simultaneously synthesized high-gain receive beams are steered in the fast-time domain over the whole swath to receive the scattered echoes. For implementation of SCORE, digital beam forming is currently considered which requires massive on-board data processing. That results in a high power consumption and creates a limitation on heat dissipation. Therefore, the application of such digital SCORE would be limited to large spacecrafts. In order to make such advanced observation modes also available for smaller SAR missions, an alternative beamforming technique is required that is compact and less power hungry. PIC based optical beam forming networks provide these features intrinsically. The advantages of PIC over conventional systems such as their broadband nature, large bandwidth and low loss capability has encouraged the Space community to exploit this novel platform in their systems.

In this work, the on-chip integrated optical building blocks such as laser, modulator, SOA and PD is utilized as a substitute for conventional digital beam forming systems. Figure 4 presents the receiver chain with a number of RF signals received from 12 antennas and then combined to 3 beams and converted to the digital domain for further processing.

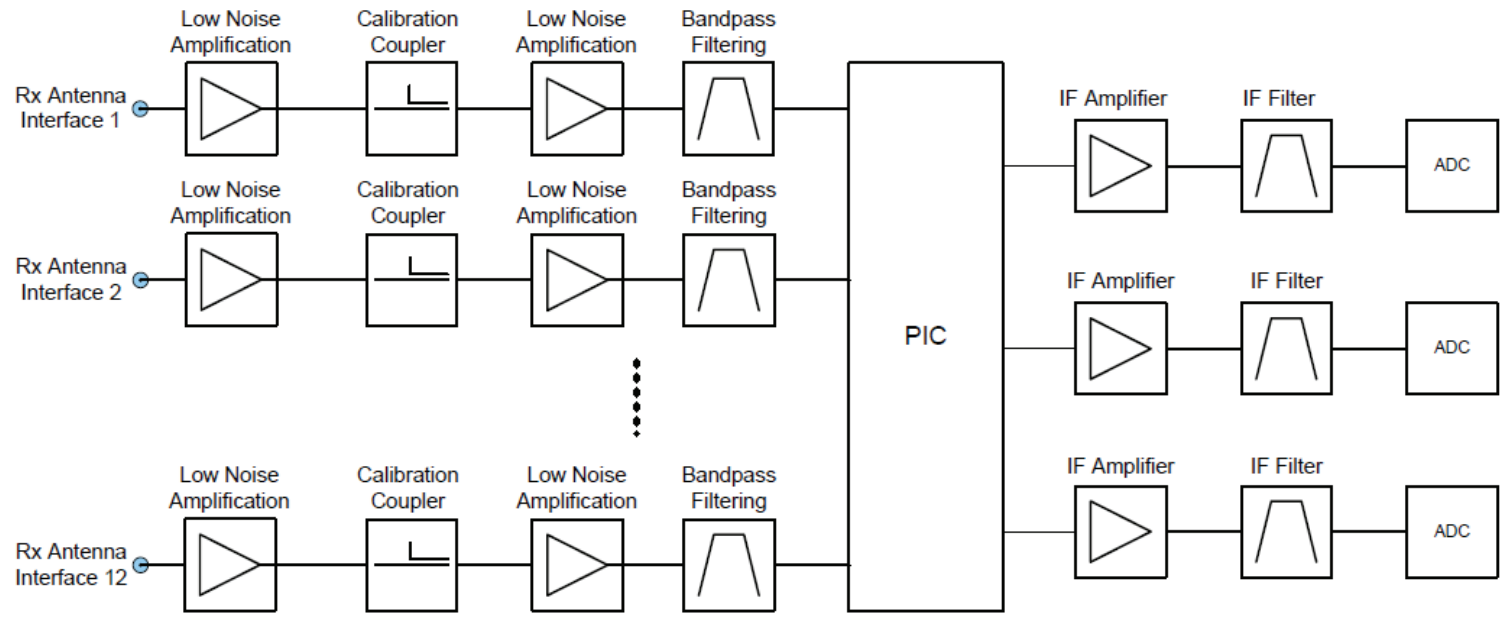

Figure 4. The receiver chain block diagram including RF front-end and PIC 
Table 2: Performance and receiver main specifications

\begin{tabular}{|c|c|}
\hline Parameter & Value \\
\hline \multicolumn{2}{|l|}{ Performance } \\
\hline Orbit Height & $>500 \mathrm{~km}$ \\
\hline Frequency band & X-band \\
\hline Swath Width (across-track) & $>30 \mathrm{~km}$, target: $>50 \mathrm{~km}$ \\
\hline Swath Length (along-track) & $>30 \mathrm{~km}$, target: $>50 \mathrm{~km}$ \\
\hline Ground Resolution & $<1.5 \mathrm{~m} \times 1.5 \mathrm{~m}$ (along-track $\times$ across-track $)$ \\
\hline \multicolumn{2}{|l|}{ Receiver } \\
\hline Functions & $\begin{array}{l}\text { - Rx protection } \\
\text { - Signal reception and amplification } \\
\text { - Rx beamforming } \\
\text { - RF signal downconversion and digitization } \\
\text { - Internal calibration signal distribution } \\
\text { Data reduction and formatting }\end{array}$ \\
\hline RF Bandwidth & $\geq 390 \mathrm{MHz}$ \\
\hline \multicolumn{2}{|c|}{ BEAMFORMER RF REQUIREMENTS } \\
\hline Function & $\begin{array}{l}\text { - Rx beamforming } \\
\text { - RF signal down-conversion }\end{array}$ \\
\hline Simultaneous beams & $\leq 3$ \\
\hline $\begin{array}{l}\text { Number of adjacent feed } \\
\text { elements to be combined for } \\
\text { one beam }\end{array}$ & $\leq 5$ \\
\hline $\begin{array}{l}\text { Number of beam steering } \\
\text { directions }\end{array}$ & $>50$, target $>100$ \\
\hline
\end{tabular}

Similar to EPFC, the PPIC here will undergo the environmental testing for demonstrating its space compliance. The other EEE devices in this phase are space grade components.

\section{LANTERN RECEIVER}

The beam tracking and steering is an important part of the laser communication terminal (LCT) which enables the LCT to effectively compensate the image motion due to the satellite movement or atmosphere turbulence. This mechanical tip-tilt compensation technique is based on the piezoelectric platform. Normally these piezo actuators spaced at $120^{\circ}$ intervals. Since expansion of an individual actuator affects both $\mathrm{x}$ and y rotation, external coordinate transformation is also required.

The problem arises when these pointing mechanisms are intended to be used in on-board mission where at one hand, the mechanical specifications are challenging and on the other hand mass and volume reduction becomes the "must" requirement to save cost. For the terminal manufacturers AIT and also maintenance cost push the technology toward more robust and compact solutions.

Photonic lantern is a passive device that multiplies the acceptance surfaces of the detection unit while keeping the insertion loss in minimum value. In order to achieve effective light coupling from the entrance aperture to the detection unit without tip-tilt mechanism, a PIC based Lantern Receiver is proposed as a powerful, integrated and multi-functional solution. The proposed PIC solution aims to provide a non-mechanical solution for beam collection over a large surface area. This area should be sufficiently large $\left(200 \times 200 \mu \mathrm{m}^{2}\right)$ to cover the full range of possible image locations from the collimation system. The operating wavelength is $1550 \mathrm{~nm}$. 
The key aspect of the PIC based solution is an array of light coupling devices. Each light coupling device is implemented as grating coupler, a well-known object which couples light incident to the surface of the PIC to a single mode waveguide. These gratings are designed such that they can be tightly packed together to reach a high fill factor. Each waveguide output is terminated with a fast (17 GHz bandwidth) and sensitive photodetector device with $0.85 \mathrm{~A} / \mathrm{W}$ responsivity.
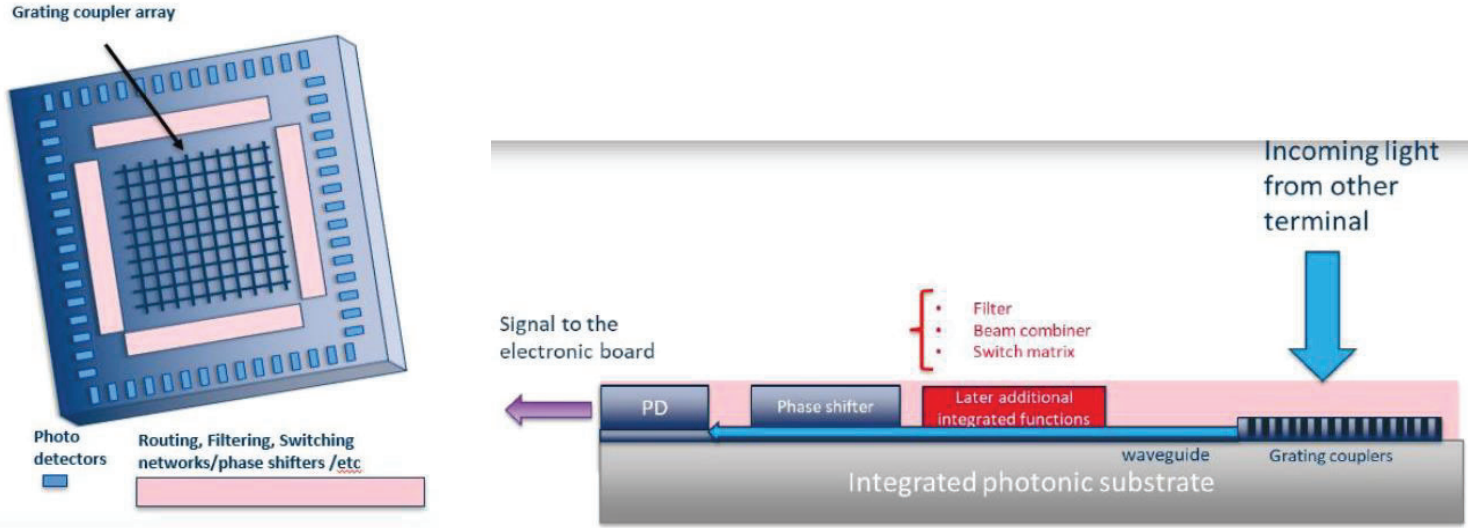

Figure 5. Left: high level schematic of the lantern receiver. Right: Side view of the photonic lantern receiver

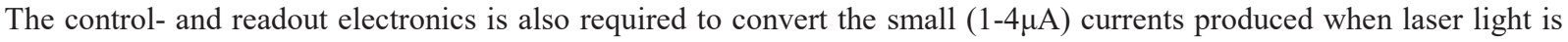
incident to the photodiodes into a voltage signal appropriate for the receiving bit error rate tester. The initial conversion is performed by a TIA. Some form of limiting amplification is also required if stable output levels are desired, so that an input of varying magnitude results in an output which is relatively constant. Multiple TIAs are needed to provide readout of the entire PD array.

\section{CONCLUSION}

In summary, we presented our innovative development activities on exploiting integrated photonics for space applications. That includes EM level Frequency down conversion from $50 \mathrm{GHz}$ to $20 \mathrm{GHz}$ for high throughput satellites, functional model of optical beam forming network in X-band for earth observation and breadboarding lantern receiver for LCTs. Advantages associated to the use of PIC in the satellites systems has been highlighted. However, further development is needed to improve maturity level of this technology.

\section{REFERENCES}

[1] V. Salvatore, et al, "Roadmap for future payloads using photonic technology. In: Third ESA Workshop on Advanced Telecom Payloads, Noordwijk, The Netherlands (2016).

[2] D. Marpaung, et al, "Integrated microwave photonics", Laser Photonics Rev. 7(4), 506-538 (2013).

[3] W. Zhang, et al, "Microwave photonic frequency conversion with high conversion efficiency and elimination of dispersion-induced power fading", IEEE Photonics Journal, (2016).

[4] Z. Tang, et al, "A Photonic Frequency Downconverter based on a Single Dual-drive Mach-Zehnder Modulator", IEEE International Topical Meeting on Microwave Photonics (MWP), (2013) 